\title{
S Research Square \\ Predictors of Undertriage after Major Trauma in a Physician-led Prehospital System: Insights of a French Registry
}

Axel BENHAMED ( $\sim$ axel.benhamed@chu-lyon.fr)

Groupement Hospitalier Edouard Herriot https://orcid.org/0000-0001-8784-5273

\section{Laurie FRATICELLI}

Vienne Hospital Centre: Centre Hospitalier de Vienne Lucien Hussel

\section{Clément CLAUSTRE}

Vienne Hospital Centre: Centre Hospitalier de Vienne Lucien Hussel

\section{Marion DOUPLAT}

Centre Hospitalier Lyon-Sud

\section{Guillaume MARCOTTE}

Groupement Hospitalier Édouard Herriot: Groupement Hospitalier Edouard Herriot

\section{Eric CESAREO}

Groupement Hospitalier Édouard Herriot: Groupement Hospitalier Edouard Herriot

\section{Amaury GOSSIOME}

Groupement Hospitalier Édouard Herriot: Groupement Hospitalier Edouard Herriot

Jean-Baptiste BOURGEOIS

Groupement Hospitalier Édouard Herriot: Groupement Hospitalier Edouard Herriot

\section{Matthieu HEIDET}

Assistance Publique Hopitaux de Paris: Assistance Publique - Hopitaux de Paris

\section{Marcel EMOND}

Hôpital de l'Enfant-Jésus: Centre hospitalier affilie universitaire de Quebec Hopital de l'Enfant-Jesus

\section{Jean-Stéphane DAVID}

Centre Hospitalier Lyon-Sud

\section{Carlos EL KHOURY}

Vienne Hospital Centre: Centre Hospitalier de Vienne Lucien Hussel

\section{Karim TAZAROURTE}

Groupement Hospitalier Édouard Herriot: Groupement Hospitalier Edouard Herriot

\section{Original research}

Keywords: trauma, undertriage, French prehospital system, mortality, registry 
Posted Date: December 30th, 2020

DOl: https://doi.org/10.21203/rs.3.rs-135822/v1

License: (c) (i) This work is licensed under a Creative Commons Attribution 4.0 International License. Read Full License 


\section{Abstract}

\section{Background}

The proper prehospital triage and transportation of patients suffering major trauma to lever 1 trauma centers is associated with better outcomes. Hence, emergency medical systems (EMS) aim is to avoid undertriage in these patients. The main objective of this study was to assess the rate and predictors of undertriage in a physician-led prehospital system.

\section{Methods}

We conducted an observational multicentric, region-wide, retrospective study based on the RESUVal Trauma-System registry, Rhône-Alpes region, France. All adults assessed by physician-led EMS units, from January 2011 to December 2017 with major trauma (Injury Severity Score (ISS) $\geq 16$ ) were included. We defined the correct-triage group as major trauma patients admitted to a level I trauma center. We performed univariate then multivariate logistic regressions with undertriage as outcome.

\section{Results}

A total of 7,110 patients were included in the registry, of whom 2,591 patients with an ISS $\geq 16$. Among these patients, 320 (12.35\%) were undertriaged. Median ISS was 25 . In-hospital mortality was $16.45 \%$ $(n=351 / 2134)$. Mid-aged patients (51-65 years old) were associated with a higher risk of undertriage than the others $(\mathrm{OR}=1.62,95 \% \mathrm{Cl} 1.15-2.28, \mathrm{p}=0.01)$. Factors associated with a lower risk of undertriage were: mechanism (fall or gunshot/stabbing wounds, 0.62 , [0.45-0.86], $p=0.01$ and 0.44, [0.22-0.9], $p=0.02$, respectively), time on-scene (over 60 minutes, $0.61,[0.38-0.95], p=0.03$ ), prehospital need for endotracheal intubation and ultrasound examination (0.53, [0.39-0.72], $p<0.001$ and $0.15,[0.08-0.29], p<0.001$ respectively). After adjusting for severity, undertriage showed a non-significant tendency toward an increased risk of mortality $(1.22,[0.8-1.89], p=0.36)$.

\section{Conclusions}

In our region-wide, physician-led prehospital system, undertriage of major trauma was not rare. The typical profile of undertriaged patients was a mid-aged male suffering from a blunt trauma, without respiratory distress or neurologic impairment, not benefiting from prehospital ultrasound examination and located close to a non-trauma center hospital.

\section{Background}

Major trauma remains a worldwide major health issue despite efforts and strategies to reduce its global burden. The World Health Organization reported that Injuries claimed 4.9 million lives in 2016 and that total deaths from injuries increased by 2.3\% between 2007 and 2017 (1). More than a quarter (29\%) of these deaths were due to road traffic injuries. 
The proper prehospital triage, and transportation, of patients suffering major trauma to level 1 trauma centers is associated with better outcomes, probably so as initial referral compared to inter-hospital transfer $(2,3)$. Therefore, emergency medical system (EMS) aim is to avoid undertriage, which defined as the transportation of patients suffering major injuries to non-level 1 trauma centers, or other acute care facilities (3).

Many definitions of undertriage exist and one of the most common is based on the Injury severity score (ISS), which is calculated at hospital discharge (4). Using this score, patients are classically considered undertriage if presenting an ISS $\geq 16$ and transported to non-level 1 trauma centers. At prehospital scene, international guidelines use algorithms or scores to predict the probability of an ISS $\geq 16$. Adherence to these guidelines adherence could reduce the risk of undertiage $(5,6)$. The American College of Surgeons Committee on Trauma consider an undertriage rate below $5 \%$ within an ideal organized trauma system (3). Therefore a recent systematic review reported within international studies an undertriage rates ranging from 1 to $71,9 \%$ (4). Few studies have investigated the characteristics of undertriaged patients, in a physician-led prehospital system. The French prehospital system is physician-led, both at call reception (for dispatch) and on scene (delivery of care). Physician-led prehospital triage system has showed its effectiveness in optimizing prehospital triage and in a regional trauma system in mountain region $(7,8)$. In a neighboring geographical area, the use of a grading system significantly decreased the risk of undertriage by $0.47(95 \% \mathrm{Cl} 0.40$ to 0.56$)$ with the ACSCOT definition of undertriage, and by $0.33(95 \% \mathrm{Cl}$ 0.26 to 0.42 ) with the TRENAU definition (7).

Our regional trauma system was implemented in 2011 and comprised composite geographical area with urban and non-urban area. We tested the hypothesis that our trauma system was mature with an undertriage rate under $5 \%$.

The main objective of this study was to therefore to assess the rate of undertriage in a physician-led prehospital system, and identify predictive factors leading to undertriage. Secondary objectives were to describe the patients who were managed for trauma by a physician-led mobile medical team (MMT) and evaluate the mortality.

\section{Methods}

\section{Study design and settings}

We conducted a multicentric retrospective study based on a trauma registry (RESUVal: Réseau des Urgences de la Vallée du Rhône) based in the Rhône Valley in France. Population area concerns 2 level I trauma centers, 1 level II and 6 level III covering a population of 3 million inhabitants (Fig. 1).

The Trauma-system registry prospectively collects prehospital and in-hospital pathways of care of adult trauma patients. Since 2011, a total of 7500 patients managed for trauma by a MMT were enrolled using a structured and standardized case report form (CRF). The pathways of care are reported by emergency 
physicians in charge of the patient from the pre-hospital management, to the emergency department, and if applicable to the intensive care unit until hospital discharge.

The pre-hospital emergency medical system in France is a two-tiered, physician-led system, and out-ofhospital emergencies are managed by the Service d'Aide Médicale d'Urgence (SAMU). Emergency calls from bystanders are centralized to the telephone number " 15 " available $24 / 7$. Then the dispatching physician can activate an MMT in critical cases (9). The MMT can either be a ground- or helicopter ambulance. MMTs are distributed throughout the region at hospital-based locations named Service Mobile d'Urgence et de Réanimation (SMUR). In suspected life-threatening injuries, both a firefighter team and an MMT are systematically dispatched to the scene. After clinical evaluation, the on-scene emergency physician and the dispatching physician determine together the most adapted care facility to refer the patient(s) according to a protocol based on Vittel criteria (7).

\section{Patients selection}

All adults managed from January 2011 to December 2017 with ISS $\geq 16$ were included in this study. Then, we defined the correct-triage group as major trauma patients admitted to one of the two regional level I trauma centers. Other patients were categorized in the undertriage group.

Patients included received written information about the objectives of the trauma-system registry in accordance with French legislation. The registry received approval from the National Commission for Liberties and Data Protection (Commission Nationale de l'Informatique et des Libertés [CNIL]) (number DE-2012-059) and received approval from the Advisory Committee on the Treatment of Research Information (Comité consultatif sur le traitement de l'information en matière de recherche [CCTIRS]).

\section{Data collection}

The registry was developed in 2011 to prospectively collect data related to the management of injured patients, from the scene to hospital admission (emergency department or intensive care unit) by the MMT physician. Research technicians provided continuous monitoring of the completeness and correctness of the database. They also collected patient outcome at hospital discharge.

The following prehospital items were prospectively recorded: age, sex, patient treatment (antiplatelet, anticoagulant), type of trauma, clinical data: systolic and diastolic arterial blood pressures, heart rate, peripheral oxygen saturation (Sp02), respiratory rate, shock index, Glasgow Coma Scale (GCS), hemoglobin test. Prehospital data care was recorded: endotracheal intubation, thoracostomy, chest tube insertion, pelvic immobilization, Focused Assessment with Sonography for Trauma (FAST). The following scores were calculated after anatomic and physiologic assessments had been completed: Abbreviated Injury Scale (AIS) (1998 version) and ISS score, M-GAP score including Mechanism, Glasgow coma scale and Age, arterial Pressure score, New Trauma and Injury Severity Score (NTRISS) and patient outcome (inhospital mortality).

\section{Outcomes of the study}


Primary aim was to assess the rate of undertriage, and predictive factors among patient epidemiology and their injury's characteristics. Secondary aims were to evaluate the association of undertriage with mortality, and to determine the maturity of the departmental trauma network, as defined by the evolution of undertriage rate from 2011 to 2017.

\section{Statistical analysis \\ Univariate analysis}

Baseline characteristics were described by frequencies and percentages for categorical variables, medians and interquartile range (IQR) for continuous variables. For categorical variables, percentages were computed among known data and the denominator was reported in tables. Comparisons between groups were assessed using the Pearson $\mathrm{Khi}^{2}$ test for categorical variables and the non-parametric Wilcoxon rank test for continuous variables. We tagged a " $X$ " in tables when statistical power was limited ( $<10$ patients per group). We also provided a temporal trend using Khi ${ }^{2}$ test for trend, referring to " $\mathrm{p}$ trend".

\section{Multiple imputations}

To perform logistic regressions, we defined candidate covariates based on the literature review and univariate analysis; age, sex, season, year of admission, type of transport, time on site, transportation time, GCS, trauma mechanism, type of accident (weapon, road, fall, other), shock index, endotracheal intubation, FAST. Among the 2, 591 included patients, the candidate covariates were $61 \%$ complete cases $(n=1583)$. To gain statistical power and reduce bias, we performed multiple imputations (MI) by generating a total of 100 datasets over 20 iterations. Regressions were calculated separately on each dataset and then combined into a single result using Rubin's rule (10). Auxiliary variables were added to optimize the imputation process (11): center level, circumstances (aggression, suicide, accident), head trauma, abdominal trauma, thoracic trauma, limb trauma, pelvis trauma, spinal trauma, prehospital administration of amines, prehospital intubation, prehospital triage, hospital endotracheal intubation, blood transfusion at shocking room, triage evaluated the shocking room, hospitalization in resuscitation unit, AIS by body zone and Glasgow Outcome Score (GOS) at discharge. In order to avoid circularity in the imputation model, a post processing has been added on the GOS so that it is not imputed for patients who are not hospitalized in intensive care. We used the mice package in $\mathrm{R}$ software (12), and observed convergence for all covariates.

\section{Sensitivity analysis}

We performed two logistic regressions with undertriage as outcome; 1 ) based on complete cases (CC) and 2) post-Ml, and calculated the variation rate $(\mathrm{VR}, \%)$ obtained as $\left.\left(100 *\left(\mathrm{OR}_{\mathrm{MI}}-\mathrm{OR}_{\mathrm{CC}}\right) / \mathrm{OR}_{\mathrm{CC}}\right)\right)$. We observed the presence of a selection bias because the patients dropped from the CC approach were not distributed completely at random (MCAR); the effects of age (especially 51-65 and $>81$ years old), the season, the gunshot circumstances and the on-scene time were characterized by the largest VR (see Additional file 1). This approach validated the relevance of the MI approach. 
We evaluated the sensitivity of the MI to the missing at random (MAR) hypothesis by using the method proposed by Héraud-Bousquet et al. (13). This method upweights imputations which are more plausible under missing not at random (MNAR). A variation rate is then computed under different deviations from the MAR hypothesis. We showed that most of the covariates were robust to a deviation to the MAR hypothesis with VR under $5 \%$. Only two variables were slightly sensitive to a deviation to the MAR hypothesis, the on scene-time and the FAST-Echo (see Additional file 2). For these two covariates, the VR was ranged between $5 \%$ and $15 \%$. Given the estimated OR, VR of $15 \%$ would not change the effect direction or significance.

\section{Multivariate logistic regressions}

A first logistic regression was used to estimate undertriage determinants as primary outcome based on 2,591 patients. The results were evaluated on each imputed datasets and pooled using the Rubin's rule.

We performed a second logistic regression based on the 1,724 patients admitted or deceased before admission in intensive care unit to estimate the effect of undertriage on mortality as secondary outcome, considering in-hospital death. This logistic regression was performed on complete cases (covariates and outcome) for $91 \%$ of the patients $(1,570 / 1,724)$. We did not use the imputed datasets from previous regression because each imputed dataset would have had different number of patients hospitalized in intensive care unit, the Rubin's rule would be impracticable. The covariates were age, sex, trauma mechanism (blunt/penetrating), pre-hospital Glasgow score, pre-hospital triage, ISS and undertriage.

\section{Results}

\section{Overall Characteristics}

From January 2011 to December 2017, 7,110 patients were screened for inclusion from the TraumaSystem registry. In total, we included 2,591 patients with an ISS $\geq 16$ for analysis. Among them, 320 (12.35\%) were defined as undertriaged (Fig. 2). The median age was 42 years old (interquartile range [IQR], 27-59) years. A total of 1,934 patients $(74.64 \%)$ were men. There was no statistically significant difference in sex or age between groups $(p=0,815$ and $p=0.0547)$. The majority of patients suffered from blunt trauma $(n=2406,94.13 \%)$. Road traffic accident was more frequent in the undertriage group than in the correct-triage ( $66.56 \%$ vs $56.14 \%, p=0.0006)$. Median on-scene time of pre-hospital management by MMTs was 36 minutes [26-51], and total prehospital time was 60 minutes [44-82]. It was shorter in the undertriage group compared to the correct-triage one ( 57.5 vs $60 \mathrm{~min}, p=0.0131)$. More than three-quarters of patients $(78.77 \%)$ were transported by ground ambulance but patients from the correct-triage group benefited more from air transportation ( $22.37 \%$ vs $12,12 \%, p=0.0002)$ (Table 1$)$. 
Table 1

Characteristics of the population

$\begin{array}{llll}\begin{array}{l}\text { Global } \\ \text { population }\end{array} & \begin{array}{l}\text { Correct triage } \\ (n=2271)\end{array} & \begin{array}{l}\text { Undertriage } \\ (n=320)\end{array} & \begin{array}{l}\text { p- } \\ \text { value }\end{array} \\ (n=2591) & & & \end{array}$

\section{Epidemiological characteristics}

\begin{tabular}{|c|c|c|c|c|}
\hline Male sex & $\begin{array}{l}1934 / 2578 \\
(75.02 \%)\end{array}$ & $\begin{array}{l}1694 / 2261 \\
(74.92 \%)\end{array}$ & $\begin{array}{l}240 / 317 \\
(75.71 \%)\end{array}$ & 0,8150 \\
\hline Age mean (IQR) & 42 [27-59] & $42[27-58]$ & $45[29-62]$ & 0,0547 \\
\hline - >65ans & $\begin{array}{l}448 / 2591 \\
(17.29 \%)\end{array}$ & $\begin{array}{l}392 / 2271 \\
(17.26 \%)\end{array}$ & $\begin{array}{l}56 / 320 \\
(17.50 \%)\end{array}$ & 0,9786 \\
\hline Season : winter & $\begin{array}{l}674 / 2591 \\
(26.01 \%)\end{array}$ & $594 / 2271(26.16 \%)$ & $\begin{array}{l}80 / 320 \\
(25.00 \%)\end{array}$ & 0,709 \\
\hline $\begin{array}{l}\text { Mechanism of injury } \\
\text { - Blunt trauma } \\
\text { - Penetrating trauma }\end{array}$ & $\begin{array}{l}2406 / 2556 \\
(94.13 \%) \\
150 / 2556 \\
(5.87 \%)\end{array}$ & $\begin{array}{l}2102 / 2239 \\
(93.88 \%) \\
137 / 2239(6.12 \%)\end{array}$ & $\begin{array}{l}304 / 317 \\
(95.90 \%) \\
13 / 317 \\
(4.10 \%)\end{array}$ & $\begin{array}{l}0,1926 \\
0,1926\end{array}$ \\
\hline $\begin{array}{l}\text { Cause of injury } \\
\text { - Road traffic accident } \\
\text { o Car occupant }\end{array}$ & $\begin{array}{l}1472 / 2563 \\
(57.43 \%) \\
602 / 1472 \\
(40.90 \%)\end{array}$ & $\begin{array}{l}1261 / 2246 \\
(56.14 \%) \\
500 / 1261 \\
(39.65 \%)\end{array}$ & $\begin{array}{l}211 / 317 \\
(66.56 \%) \\
102 / 211 \\
(48.34 \%)\end{array}$ & $\begin{array}{l}0,0006 \\
0,0214 \\
0,1102\end{array}$ \\
\hline \multirow{4}{*}{$\begin{array}{l}\text { o Motorcycle } \\
\text { o Bicycle } \\
\text { o Pedestrian } \\
\text { - Fall } \\
\text { - Suicide } \\
\text { - Gunshot/stab wounds }\end{array}$} & $\begin{array}{l}500 / 1472 \\
(33.97 \%)\end{array}$ & $\begin{array}{l}439 / 1261 \\
(34.81 \%)\end{array}$ & $\begin{array}{l}61 / 211 \\
(28.91 \%)\end{array}$ & $\begin{array}{l}0,1252 \\
0,0059\end{array}$ \\
\hline & $\begin{array}{l}124 / 1472 \\
(8.42 \%)\end{array}$ & $\begin{array}{l}100 / 1261(7.93 \%) \\
218 / 1261\end{array}$ & $\begin{array}{l}24 / 211 \\
(11.37 \%)\end{array}$ & 0,0901 \\
\hline & $\begin{array}{l}238 / 1472 \\
(16.17 \%)\end{array}$ & $(17.29 \%)$ & $\begin{array}{l}20 / 211 \\
(9.48 \%)\end{array}$ & \\
\hline & $\begin{array}{l}542 / 2563 \\
(21.15 \%)\end{array}$ & $\begin{array}{l}48 / 22246 \\
(21.68 \%)\end{array}$ & $\begin{array}{l}55 / 317 \\
(17.35 \%)\end{array}$ & $\begin{array}{l}X \\
0,0798\end{array}$ \\
\hline - Other & $\begin{array}{l}195 / 2243 \\
(8.69 \%)\end{array}$ & $108 / 2246(4.81 \%)$ & $\begin{array}{l}21 / 291 \\
(7.22 \%)\end{array}$ & \\
\hline & $\begin{array}{l}117 / 2563 \\
(4.56 \%)\end{array}$ & $\begin{array}{l}390 / 2246 \\
(17.36 \%)\end{array}$ & $\begin{array}{l}9 / 317 \\
(2.84 \%)\end{array}$ & \\
\hline & $\begin{array}{l}432 / 2563 \\
(16.86 \%)\end{array}$ & & $\begin{array}{l}42 / 317 \\
(13.25 \%)\end{array}$ & \\
\hline
\end{tabular}




\begin{tabular}{|c|c|c|c|c|}
\hline & $\begin{array}{l}\text { Global } \\
\text { population } \\
(n=2591)\end{array}$ & $\begin{array}{l}\text { Correct triage } \\
(n=2271)\end{array}$ & $\begin{array}{l}\text { Undertriage } \\
(n=320)\end{array}$ & $\begin{array}{l}\mathrm{p}- \\
\text { value }\end{array}$ \\
\hline \multirow{2}{*}{$\begin{array}{l}\text { Treatment } \\
\text { - Anti-platelet } \\
\text { - Anticoagulant }\end{array}$} & $\begin{array}{l}158 / 2591 \\
(6.10 \%)\end{array}$ & & $\begin{array}{l}21 / 320 \\
(6.56 \%)\end{array}$ & \multirow{2}{*}{$\begin{array}{l}0,8056 \\
0,5021\end{array}$} \\
\hline & \multicolumn{2}{|l|}{$\begin{array}{l}85 / 2591 \\
(3.28 \%)\end{array}$} & $\begin{array}{l}13 / 320 \\
(4.06 \%)\end{array}$ & \\
\hline \multicolumn{5}{|l|}{ Pre-hospital timing and transport } \\
\hline On scene time (min) & $36[26-51]$ & $36[26-52]$ & 34 [24-48.5] & 0,0086 \\
\hline $\begin{array}{l}\text { Transit time from scene to } \\
\text { hospital }\end{array}$ & $20[13-30]$ & $20[13-30]$ & $21[14-30]$ & 0,8276 \\
\hline Total pre-hospital time & 60 [44-82] & $60[45-83.25]$ & $\begin{array}{l}57.5[41- \\
75.25]\end{array}$ & 0,0131 \\
\hline \multirow{2}{*}{$\begin{array}{l}\text { Transport } \\
\text { - Air transport } \\
\text { - Ground transport }\end{array}$} & $\begin{array}{l}550 / 2591 \\
(21.23 \%)\end{array}$ & $\begin{array}{l}508 / 2271 \\
(22.37 \%)\end{array}$ & $\begin{array}{l}42 / 320 \\
(13.12 \%)\end{array}$ & \multirow{2}{*}{$\begin{array}{l}0,0002 \\
0,0002\end{array}$} \\
\hline & $\begin{array}{l}2041 / 2591 \\
(78.77 \%)\end{array}$ & $\begin{array}{l}1763 / 2271 \\
(77.63 \%)\end{array}$ & $\begin{array}{l}278 / 320 \\
(86.88 \%)\end{array}$ & \\
\hline \multicolumn{5}{|l|}{ Pre-hospital clinical data } \\
\hline Heart rate (beat per minute) & $90[75-106]$ & $90[75-106]$ & $90[75-108]$ & 0,8131 \\
\hline Blood pressure $(\mathrm{mmHg})$ & $125[109-142]$ & $125[109-142.5]$ & $124[109-$ & 0,5273 \\
\hline - Systolic & $78[65-90]$ & 78 [65-90] & \multirow{2}{*}{$\begin{array}{l}75[65-87] \\
92.3[80- \\
103.3]\end{array}$} & 0,1246 \\
\hline $\begin{array}{l}\text { - Diastolic } \\
\text { - Mean }\end{array}$ & $\begin{array}{l}93.3[80.6- \\
106.7]\end{array}$ & 93.3 [80.3-107.3] & & 0,2084 \\
\hline Respiratory rate (breaths/min) & 18 [15-20] & $18[15-20]$ & 20 [16-20.25] & 0,0062 \\
\hline SpO2 & 98 [94-99] & $98[94-100]$ & 97 [94-99] & 0,0147 \\
\hline \multirow{2}{*}{$\begin{array}{l}\text { Shock index } \\
\text { - Shock index } \geq 0.90\end{array}$} & $\begin{array}{l}0.706[0.569- \\
0.894]^{-}\end{array}$ & $\begin{array}{l}0.705[0.567- \\
0.896]\end{array}$ & $\begin{array}{l}0.708 \\
{[0.577-} \\
0.889]\end{array}$ & 0,6781 \\
\hline & $\begin{array}{l}566 / 2317 \\
(24.43 \%)\end{array}$ & $\begin{array}{l}499 / 2034 \\
(24.53 \%)\end{array}$ & $\begin{array}{l}67 / 283 \\
(23.67 \%)\end{array}$ & \\
\hline
\end{tabular}

Statistics presented: $\mathrm{n}(\%)$; median [IQR] 


\begin{tabular}{|c|c|c|c|c|}
\hline & $\begin{array}{l}\text { Global } \\
\text { population } \\
(n=2591)\end{array}$ & $\begin{array}{l}\text { Correct triage } \\
(n=2271)\end{array}$ & $\begin{array}{l}\text { Undertriage } \\
(n=320)\end{array}$ & $\begin{array}{l}\mathrm{p}- \\
\text { value }\end{array}$ \\
\hline \multirow{3}{*}{$\begin{array}{l}\text { Glasgow score } \\
-<15 \\
-\leq 8\end{array}$} & $14[7-15]$ & $14[7-15]$ & 15 [10.25-15] & 0,0020 \\
\hline & \multirow{2}{*}{$\begin{array}{l}1303 / 2441 \\
(53.38 \%) \\
\\
696 / 2441 \\
(28.51 \%)\end{array}$} & $\begin{array}{l}1167 / 2143 \\
(54.46 \%)\end{array}$ & $\begin{array}{l}136 / 298 \\
(45.64 \%)\end{array}$ & \multirow{2}{*}{$\begin{array}{l}0,0052 \\
0,0051\end{array}$} \\
\hline & & $\begin{array}{l}632 / 2143 \\
(29.49 \%)\end{array}$ & $\begin{array}{l}64 / 298 \\
(21.48 \%)\end{array}$ & \\
\hline \multirow{2}{*}{$\begin{array}{l}\text { Hemoglobin test on scene }(\mathrm{g} / \mathrm{dl}) \\
-\leq 13\end{array}$} & 13.5 [12-14.8] & 13.5 [12-14.9] & \multirow{2}{*}{$\begin{array}{l}13.1[11.6- \\
14.7] \\
111 / 226 \\
(49.12 \%)\end{array}$} & \multirow{2}{*}{$\begin{array}{l}0,0558 \\
0,0410\end{array}$} \\
\hline & $\begin{array}{l}912 / 2144 \\
(42.54 \%)\end{array}$ & $\begin{array}{l}801 / 1918 \\
(41.76 \%)\end{array}$ & & \\
\hline \multicolumn{5}{|l|}{ Pre-hospital management } \\
\hline Endotracheal intubation & $\begin{array}{l}949 / 2353 \\
(40.33 \%)\end{array}$ & $\begin{array}{l}875 / 2071 \\
(42.25 \%)\end{array}$ & $\begin{array}{l}74 / 282 \\
(26.24 \%)\end{array}$ & $\dot{0} 00001$ \\
\hline Thoracostomy & $\begin{array}{l}60 / 2337 \\
(2.57 \%)\end{array}$ & $55 / 2056(2.68 \%)$ & $\begin{array}{l}5 / 281 \\
(1.78 \%)\end{array}$ & $x$ \\
\hline Chest tube insertion & $\begin{array}{l}28 / 2337 \\
(1.20 \%)\end{array}$ & 26/2056 (1.26\%) & $\begin{array}{l}2 / 281 \\
(0.71 \%)\end{array}$ & $x$ \\
\hline Pelvic immobilization & $\begin{array}{l}326 / 2336 \\
(13.96 \%)\end{array}$ & $\begin{array}{l}284 / 2057 \\
(13.81 \%)\end{array}$ & $\begin{array}{l}42 / 279 \\
(15.05 \%)\end{array}$ & 0,6369 \\
\hline $\begin{array}{l}\text { Focused assesment with } \\
\text { sonography (FAST) }\end{array}$ & $\begin{array}{l}400 / 2333 \\
(17.15 \%)\end{array}$ & $\begin{array}{l}391 / 2053 \\
(19.05 \%)\end{array}$ & $\begin{array}{l}9 / 280 \\
(3.21 \%)\end{array}$ & $x$ \\
\hline
\end{tabular}

A total of 566 patients $(17.95 \%)$ had a shock index $\geq 0.9$ and 696 (28.51\%) presented with major head trauma (GSC $\leq 8)$.

Pre-hospital management is described in Table 1. The most frequent intervention was endotracheal intubation $(40.33 \%)$ which was more frequent in the correct triage group $(42.25 \%$ vs $26.24 \%, p<0.0001)$. Table 2 reports injuries characteristics and outcome of injured patients. ISS was higher in correct-triaged patient $(25$ [19-34] vs 21.5 [17-29], $p<0.0001))$. The most frequent severely injured (AIS $\geq 3$ ) body areas were head $(53.57 \%)$ and thorax $(54.11 \%)$. In-hospital mortality was $16.45 \%(n=351 / 2134)$ with no statistical difference between groups $(16.22 \%$ vs $18.30 \%, p=0.4730)$ 
Table 2

Injuries characteristics and outcome

$\begin{array}{llll}\begin{array}{l}\text { Global } \\ \text { population }\end{array} & \begin{array}{l}\text { Correct } \\ \text { triage }\end{array} & \begin{array}{l}\text { Undertriage } \\ (n=320)\end{array} & \begin{array}{l}\text { p- } \\ \text { value }\end{array} \\ (n=2591) & (n= & & \\ & 2271) & & \end{array}$

\section{Trauma scoring}

ISS: Injury severity score

$\begin{array}{llll}25[18-34] & 25[19- & 21.5[17- & < \\ & 34]^{17} & 29] & 0.0001\end{array}$

MGAP: Mechanism, Glasgow coma scale, Age, $\quad 24[20-27] \quad 24[20-\quad 25[21-28] \quad 0.0294$ arterial Pressure

$-<23$

849

$(37.68 \%)$
27]

754 (38.06\%)
95

$(34.93 \%)$

\section{Severe injuries (AIS $\geq 3$ ) by body region}

\begin{tabular}{|c|c|c|c|c|}
\hline 1: Head & $\begin{array}{l}1388 \\
(53.57 \%)\end{array}$ & $\begin{array}{l}1224 \\
(53.90 \%)\end{array}$ & $\begin{array}{l}164 \\
(51.25 \%)\end{array}$ & 0.4071 \\
\hline 2: Face & $128(4.94 \%)$ & $\begin{array}{l}119 \\
(5.24 \%)\end{array}$ & $9(2.81 \%)$ & $x$ \\
\hline 3: Thorax & $\begin{array}{l}1402 \\
(54.11 \%)\end{array}$ & $\begin{array}{l}1225 \\
(53.94 \%)\end{array}$ & $\begin{array}{l}177 \\
(55.31 \%)\end{array}$ & 0.6884 \\
\hline 4: Abdomen & $\begin{array}{l}569 \\
(21.96 \%)\end{array}$ & $\begin{array}{l}517 \\
(22.77 \%)\end{array}$ & $\begin{array}{l}52 \\
(16.25 \%)\end{array}$ & 0.0104 \\
\hline 5: Upper/lower extremities & $\begin{array}{l}923 \\
(35.62 \%)\end{array}$ & $\begin{array}{l}834 \\
(36.72 \%)\end{array}$ & $\begin{array}{l}89 \\
(27.81 \%)\end{array}$ & $\begin{array}{l}< \\
0.0023\end{array}$ \\
\hline 6: Skin & $23(0.89 \%)$ & $\begin{array}{l}20 \\
(0.88 \%)\end{array}$ & $3(0.94 \%)$ & $x$ \\
\hline \multicolumn{5}{|l|}{ Outcome } \\
\hline Intensive care unit & $\begin{array}{l}2011 \\
(81.88 \%)\end{array}$ & $\begin{array}{l}1749 \\
(81.24 \%)\end{array}$ & $\begin{array}{l}262 \\
(86.47 \%)\end{array}$ & 0.0328 \\
\hline In-hospital death & $\begin{array}{l}351 \\
(16.45 \%)\end{array}$ & $\begin{array}{l}308 \\
(16.22 \%)\end{array}$ & $\begin{array}{l}43 \\
(18.30 \%)\end{array}$ & 0.4730 \\
\hline \multirow{2}{*}{$\begin{array}{l}\text { NTRISS: Death risk according to New Trauma } \\
\text { and Injury Severity Score }\end{array}$} & 0.062 & 0.063 & 0.057 & \multirow{2}{*}{$<.0006$} \\
\hline & $\begin{array}{l}{[0.02-} \\
0.227]\end{array}$ & $\begin{array}{l}{[0.02-} \\
0.244]\end{array}$ & $\begin{array}{l}{[0.017-} \\
0.122]\end{array}$ & \\
\hline
\end{tabular}

Statistics presented: $\mathrm{n}(\%)$; median [IQR]

\section{Primary outcome}


In total, 320 patients (12.53\%) were undertriaged. The results of univariate and multivariate analyses of global population characteristics are shown in Fig. 3. Gender was not predictive of undertriage. Compared to women, male had an $\mathrm{OR}=1.04(95 \% \mathrm{Cl}[0.79-1.37], \mathrm{p}=0.76)$. In the multivariate logistic regression analysis, the mid-aged patients (51-65 years old) were associated with a higher risk of undertriage than others $(\mathrm{OR}=1.62,95 \% \mathrm{Cl} 1.15-2.28, \mathrm{p}=0.01)$. Patients older than 80 years showed a tendency toward an increased risk of undertriage but it was not statistically significative $(O R=1.58,95 \% \mathrm{Cl} 0.93-2.68, p=$ 0.76). The cause of injury was a predictive factor for undertriage, compared to road traffic accident, patient suffering from fall or gunshot/stabbing wounds were less undertriaged than others $(O R=0.62$, $95 \% \mathrm{Cl} 0.45-0.86, \mathrm{p}=0.01$ and $\mathrm{OR}=0.44,95 \% \mathrm{Cl} 0.22-0.90, \mathrm{p}=0.02$, respectively). In univariate analysis, $\mathrm{GSC} \leq 8$ was a protective factor for undertriage $(\mathrm{OR}=0.60,95 \% \mathrm{Cl} 0.44-0.81, \mathrm{p}=.0 .001)$. On-scene time was a predictive factor for undertriage: over 60 minutes, patients were less likely to be undertriaged than those in shorter times $(\mathrm{OR}=0.61,95 \% \mathrm{Cl} 0.39-0.95, \mathrm{p}=0.03)$. Also, patients who needed endotracheal intubation or benefited from a FAST were less undertriaged than others $(O R=0.53,95 \% \mathrm{Cl} 0.39-0.72, p<$ 0.001 and $\mathrm{OR}=0.15,95 \% \mathrm{Cl} 0.08-0.29, \mathrm{p}<0.001$ respectively).

\section{Secondary outcomes}

Although close to being significantly associated with a reduced risk of death in univariate analysis $(\mathrm{OR}=$ $0.72,95 \% \mathrm{Cl} 0.52-1.01, \mathrm{p}=0.06)$, undertriage was not associated with a reduction of mortality in multivariate analysis $(\mathrm{OR}=1.22,95 \% \mathrm{Cl} 0.80-1.89, \mathrm{p}=0.36)$. Neither were gender, nor trauma mechanism. Mortality was higher in patients suffering from major head trauma (GSC $\leq 8)$, OR $=3.31(95 \% \mathrm{Cl} 2.33-$ 4.69) $\mathrm{p}<0.001$. Also, ISS was predictive for mortality $(\mathrm{OR}=1.43,95 \% \mathrm{Cl} 1.33-1.54, \mathrm{p}<0.001)$. NTRISS (New Trauma and Injury Severity Score) score predicted a lower 30-days mortality for undertriaged patient $(0.057[0.017 ; 0.122]$ vs $0.063[0.02 ; 0.244], p=0.0006)$

Figure 4 shows the annual triage of patients severely injured included in this study. There were 270 patients included in 2011 with a 16.3\% $(n=44)$ undertriage rate. In 2017, among 440 patients included, $11.4 \%(n=50)$ were undertriaged. There was each year more patients included but no significant change in the undertriage rate from 2011 to 2017 ( $p$-trend $=0.65$ ). Minimum was in $2013(7.65 \%)$ and maximum in 2016 (17.24\%).

\section{Discussion}

In our study, undertriage rate was $12.35 \%$ among patients included in our regional database as they received first care at non-level I centers. This result is above the recommendations of less than $5 \%$ as set by the ACSCOT (3). Many European and American studies reported similar or even higher undertriage rate $(4,14,15,16)$. In a comparable health system also benefiting from physician on scene and medical dispatching, French authors reported higher undertriage rate about $18 \%$ (7). Voskens et al. reported a $21.6 \%(95 \% \mathrm{Cl}, 18.0-25.7)$ rate of undertriage. In their study, allocation of trauma patients to the appropriate level of trauma care was guided by the Dutch Field Triage Protocol and their ambulance care system was nursebased (17). 
In paramedic-led prehospital systems, undertriage was higher: Schellenberg and al reported $16 \%$ undertriage (21), Xiang and al reported 34\% (14).

Our findings showed no association between undertriage and in-hospital mortality, contrary to other studies $(6,18,19)$. Probably because a large amount of undertriaged patients $(n=202,63.1 \%)$ were admitted in the center considered as level II because there is no neurosurgery department nor thoracic surgery department but yet offers large 24-hour in-house resources (intensive care, general and orthopedic surgeons, emergency physicians, radiology). Some of these patients were secondarily transferred to a level 1 center but no data were available about the reason of such transfer, or about vital status at secondary hospital discharge. Pickering et al. reported no difference in outcomes for direct referral to a trauma center versus initial triage to a local hospital in their systematic review (19).

In our study, penetrating trauma were rare, counting for only $4.56 \%$. This is consistent with other French and European series. An interesting finding was that compared to road traffic accident, fall and gunshot/stab wounds were protective for undertriage. Schellenberg et al. also reported higher risk for undertriage following blunt trauma, especially motor vehicle collisions (21). When the gravity is not as obvious as these penetrating injuries, we should search for other predictors to guide field triage. In our study, any age showed a tendency toward higher risk of undertriage, except age of 51 to 65 years that was a predictive factor of undertriage. Nakahara et al. found the same result in the group 45-54 years (22). On the opposite, most studies reported higher age cut-off for undertriage risk: $\geq 55$ years (14), $\geq$ 65 years $(6,18), \geq 80$ years $(23)$. In our study, the field triage criteria for level I were: respiratory failure (Sp02 $<90 \%$ despite oxygen supply), hypotension (MAP $<100 \mathrm{mmHg}$ despite $1000 \mathrm{~mL}$ of fluid resuscitation) GCS $\leq 8$, use of vasopressor or blood transfusion. Hence, a possible explanation is that in our trauma system, neither age ,nor comorbidity excluded referral at level 1 centers. Indeed, previous studies have reported improved outcomes for elderly patients admitted in higher levels of trauma care (24-26) Some authors even suggested that treating severely injured elderly trauma patients in designated geriatric trauma centers or units may be associated with statistically significant gains in likelihood of survival $(27,28)$. Bradburn et al. assessed a specific protocol to identify high-risk geriatric injured patients which lead to significant reduced mortality (29)

Several studies found that patients suffering from head injury were more affected by undertriage $(14,15)$. We did not report that trend. On the opposite, GSC $\leq 8$ was a protective factor for undertriage. So was the need for endotracheal intubation regardless of the GSC.

A new finding of this study is that patient who benefited from a FAST were less undertriaged regardless of hemodynamic condition. Ultrasound-based clinical pathways might enhance the speed of injured patient assessment. But effectiveness of ultrasound-based clinical pathways to triage algorithms lacks evidence and its benefit remains unclear (30). In our study, FAST was not mandatory, not integrated in the field triage algorithm and results were not available. We ignored if triage decision was or not based on FAST results. We hypothesized that when an effusion was detected, physicians would consider patients to require higher care (e.G. a level 1 center referral). Another hypothesis considered that physicians 
performing FAST were more experienced with better assessment capacities than other colleagues. In our cohort, ultrasonography was performed in $2.7 \%$ cases in 2011 while it was in $34.9 \%$ in 2017 . It may contribute to lower undertriage rate in the next years. Further explorations are needed to integrate FAST in our field triage algorithm and assess its performance.

Air transport also appeared to be protective against undertriage compared to ground transport regardless of severity (ISS) and transit time from scene to hospital. It might be explained by highest availability of helicopter emergency medical service (HEMS) in level I center. Therefore, we assumed that physicians using HEMS tended to facilitate the referral of patients to their level 1 center instead. Nevertheless, we showed no influence of transport on in-hospital mortality. Other studies did no report strong evidence of any benefice of HEMS on in-hospital mortality among injured patients $(31,32,33)$

Fukuma et al. showed that physiological variables plus lactate on-scene significantly increased the predictive value for immediate intervention for hemorrhage (Area Under the Curve $0.882,95 \% \mathrm{Cl} 0.839-$ 0.925 ) compared to that using physiological variables only (AUC $0.837,95 \% \mathrm{Cl} 0.787-0.887, \mathrm{p}=0.0073$ ) (34). It may be interesting to investigate the role of on-scene lactate measuring during field triage and its impact on undertriage.

One strength of our study was to use ISS score (16 or more) to assess trauma severity and undertriage, as most of the studies to date, and as recommended by the American College of Surgeons (3). In 2019, a systematic review reported four categories to define undertriage: based on ISS, formula for mis-triage (1sensitivity), need for life-saving emergency intervention and patients triaged to a non-trauma center (4). This statement led to significant heterogeneity that complicates comparison across studies. The standardization of undertriage definition is a priority to estimate the true rate among different studies and clarify the role of triage tools. Also, French and American level's center categorization in trauma system are different. In France, levels are approved by the regional agency for health and their definition is common for every region in the country, whereas American trauma categories can vary from state to state. On the opposite, the American college of surgeons (ACS) does not designate trauma centers but it verifies the presence of the resources listed in Resources for Optimal Care of the Injured Patient (3). Finally, ACS reports five levels while French system has three.

This study has several limitations. First, exact locations of the on-scene trauma were not available in the database. Thus we could not evaluate the distance towards the nearest trauma center and definitive destination. Whether the physician decision was based on the triage algorithm, their own clinical judgment or any other element was not reported. Some missing data could also have influenced the destination such as chronic conditions, result of the FAST, trauma center overcrowding.

\section{Conclusions}

In our region-wide, physician-led prehospital system, undertriage of major trauma concerned $12.35 \%$ of major traumas. The typical profile of undertriaged patients was a mid-aged male suffering from a blunt 
trauma, without respiratory distress or neurologic impairment, not benefiting from prehospital ultrasound examination and located close to a non-trauma center hospital.

Despite the creation of our regional trauma system, and the physician-led process of on-scene care delivery and adjusted referral of patients, the undertriage rate has not significantly decreased between 2011 and 2017. The need for triage tools that reinforce medical expertise is probably necessary to improve the quality of care.

\section{Abbreviations}

AIS: Abbreviated injury scale

EMS: Emergency medical system

FAST: Focused assessment with sonography for trauma

GSC: Glasgow coma scale

ISS: Injury severity score

MMT: Mobile medical team

SAMU: Service d'aide médicale d'urgence

SMUR : Service mobile d'urgence et de réanimation

\section{Declarations}

\section{Ethics approval and consent to participate}

The Trauma-System registry received approval from the National Commission for Liberties and Data Protection (Commission Nationale de l'Informatique et des Libertés, CNIL, reference number DE-2012059) and received approval from the Advisory Committee on the Treatment of Research Information (Comité consultatif sur le traitement de l'information en matière de recherche scientifique, CCTIRS, reference number: 15.368). In accordance with the French law, all patients included were informed of their participation and could oppose the use of their data if they wished.

\section{Consent for publication}

Not applicabale

\section{Availability of data and materials}


Data are available upon reasonnable request.

\section{Competing interests}

Authors do not declare any conflict of interest for this original article

\section{Funding}

Authors did not receive any funding for this work.

The Trauma-System registry is funded by the Regional Agency for health (ARS, Agence Régionale de Santé Auvergne Rhône-Alpes) and coordinated by the RESCUe-RESUVal.

This research did not receive any specific grant.

\section{Authors' contributions}

Axel BENHAMED: Literature search, study design, data collection, data interpretation, writing

Laurie FRATICELLI: Study design, data analysis, critical revision

Clément CLAUSTRE: Data analysis, Critical revision

Marion DOUPLAT: Critical revision

Guillaume MARCOTTE: Critical revision

Eric CESAREO: Critical revision

Amaury GOSSIOME: Critical revision

Jean-Baptiste BOURGEOIS: Critical revision

Matthieu HEIDET: Critical revision

Marcel EMOND: Critical revision

Jean-Stéphane DAVID: Data collection, study design and critical revision

Carlos EL KHOURY: Critical revision

Karim TAZAROURTE: Literature search, study design, data collection, data interpretation, writing 


\section{Acknowledgements}

We thank Julie Freyssenge, health geographer $\mathrm{PhD}$, for providing the map of trauma center. We thank the local investigators of the Trauma-System Registry: Antoine Blum (SMUR 26, Saint Vallier), Bastien Anette (SMUR 69, Villefranche-sur-Saone), Djamel Bekka (SMUR 07, Privas), Eric Cesareo (SMUR 69, Lyon), Marine Demarquet (SMUR 01, Bourg-en-Bresse), Patricia Trinquet (SMUR 38, Vienne), Stéphane Blain (SMUR 01, Ambérieu), Thomas Paita (SMUR 38, Bourgoin-Jallieu), Johan Restier (SMUR 26, Valence), Olivier Debas (SMUR 01, Belley), Raphael Brilland (SMUR 69, Tarare), Nicolas Roumestand (SMUR 26, Montélimar), Guillaume Marcotte (HEH), Jean-Stéphane David (CHLS), Etienne Javouhey (HFME), Loïs Grattier (CH Valence), Frédéric Verbois (CH Villefranche-sur-Saone), Patrice Serre (CH Bourg-en-Bresse).

\section{References}

1. Roth G, Abate D, Abate KH, Abay S, Abbafati C, Abbasi N, Abbastabar H, Abd-Allah D, Abdela D, Abdelalim A et al. Global, regional, and national age-sex-specific mortality for 282 causes of death in 195 countries and territories, 1980-2017: a systematic analysis for the Global Burden of Disease Study 2017. Lancet. 2018;392:1736-88.

2. Garwe T, Cowan LD, Neas BR, Sacra JC, Albrecht RM. Directness of Transport of Major Trauma Patients to a Level I Trauma Center: A Propensity-Adjusted Survival Analysis of the Impact on ShortTerm Mortality. J Trauma Acute Care Surg. 2011;70:1118-27.

3. American College of Surgeons, Committee on Trauma. Resources for optimal care of the injured patient. Chicago, III.: American College of Surgeons, Committee on Trauma; 2014.

4. Najafi Z, Abbaszadeh A, Zakeri H, Mirhaghi A. Determination of mis-triage in trauma patients: a systematic review. Eur J Trauma Emerg Surg. 2019;45:821-39.

5. Tignanelli CJ, Vander Kolk WE, Mikhail JN, Delano MJ, Hemmila MR. Noncompliance with American College of Surgeons Committee on Trauma recommended criteria for full trauma team activation is associated with undertriage deaths. J Trauma Acute Care Surg. 2018;84:287-94.

6. Polites SF, Leonard JM, Glasgow AE, Zielinski MD, Jenkins DH, Habermann EB. Undertriage after severe injury among United States trauma centers and the impact on mortality. Am J Surg. 2018;216:813-8.

7. Bouzat P, Ageron FX, Brun J, Levrat A, Berthet M, Rancurel E, Thouret JM, Thony F, Arvieux C, Payen JF. A regional trauma system to optimize the pre-hospital triage of trauma patients. Crit Care. 2015;19:111.

8. Bouzat P, David JS, Tazarourte K. French regional trauma network: the Rhone-Alpes example. $\mathrm{Br} \mathrm{J}$ Anaesth. 2015;114:1004-5.

9. Adnet F, Lapostolle F. International EMS Systems: France. Resuscitation. 2004;63:7-9.

10. Heymans MW, Eekhout I. Applied missing data analysis with SPSS and (R)Studio. https://bookdown.org/mwheymans/bookmi/. Accessed 10 September 2020. 
11. Collins LM, Schafer JL, Kam CM. A comparison of inclusive and restrictive strategies in modern missing data procedures. Psychol Methods. 2001;6:330-51.

12. Van Buuren S , Groothuis Oudshoorn K. Mice: Multivariate Imputation by Chained Equations in R. J Stat Softw . https://www.jstatsoft.org/article/view/v045i03. Accessed 10 September 2020.

13. Héraud Bousquet V, Larsen C, Carpenter J, Desenclos JC, Le Strat Y. Practical considerations for sensitivity analysis after multiple imputation applied to epidemiological studies with incomplete data. BMC Med Res Methodol. 2012;12:73.

14. Xiang H, Wheeler KK, Groner JI, Shi J, Haley KJ. Undertriage of major trauma patients in the US emergency departments. Am J Emerg Med. 2014;32:997-1004.

15. Nordgarden T, Odland P, Guttormsen AB, Ugelvik KS. Undertriage of major trauma patients at a university hospital: a retrospective cohort study. Scand J Trauma Resusc Emerg . 2018;26:64.

16. Van Rein EAJ, Van Der Sluijs R, Houwert RM, Gunning AC, Lichtveld RA, Leenen LPH, Van Heijl M. Effectiveness of prehospital trauma triage systems in selecting severely injured patients: Is comparative analysis possible? Am J Emerg Med. 2018;36:1060-9.

17. Voskens FJ, Van Rein EAJ, Van Der Sluijs R, Houwert RM, Lichtveld RA, Verleisdonk EJ, Segers M, Van Olden G, Dijkgraaf M, Leenen LPH. Accuracy of prehospital triage in selecting severely injured trauma patients. JAMA Surg. 2018;153:322-327.

18. Rogers A, Rogers FB, Schwab CW, Bradburn E, Lee J, Wu D, Miller JA. Increased mortality with undertriaged patients in a mature trauma center with an aggressive trauma team activation system. Eur J Trauma Emerg Surg. 2013;39:599-603.

19. Haas B, Gomez D, Zagorski B, Stukel TA, Rubenfeld GD, Nathens AB. Survival of the fittest: the hidden cost of undertriage of major trauma. J Am Coll Surg. 2010;211:804-11.

20. Pickering A, Cooper K, Harnan S, Sutton A, Mason S, Nicholl J. Impact of prehospital transfer strategies in major trauma and head injury: Systematic review, meta-analysis, and recommendations for study design. J Trauma Acute Care Surg. 2015;78:164-77.

21. Schellenberg M, Benjamin E, Bardes JM, Inaba K, Demetriades D. Undertriaged trauma patients: Who are we missing?. J Trauma Acute Care Surg. 2019;87:865-9.

22. Nakahara S, Matsuoka T, Ueno M, Mizushima Y, Ichikawa M, Yokota J, Yoshida K. Predictive Factors for Undertriage Among Severe Blunt Trauma Patients: What Enables Them to Slip Through an Established Trauma Triage Protocol?. J Trauma Acute Care Surg. 2010;68:1044-51.

23. Amoako J, Evans S, Brown NV, Khaliqdina S, Caterino JM. Identifying Predictors of Undertriage in Injured Older Adults After Implementation of Statewide Geriatric Trauma Triage Criteria. Acad Emerg Med. 2019;26:648-56.

24. Goodmanson NW, Rosengart MR, Barnato AE, Sperry JL, Peitzman AB, Marshall GT. Defining geriatric trauma: when does age make a difference?. Surgery. 2012;152:668-75.

25. Shifflette VK, Lorenzo M, Mangram AJ, Truitt MS, Amos JD, Dunn EL. Should age be a factor to change from a level II to a level I trauma activation?. J Trauma Acute Care Surg. 2010;69:88-92. 
26. Rogers A, Rogers F, Bradburn E, Krasne M, Lee J, Wu D, Edavettal M, Horst M. Old and undertriaged: a lethal combination. Am Surg. 2012;78:711-715.

27. Pracht EE, Langland Orban B, Flint L. Survival Advantage for Elderly Trauma Patients Treated in a Designated Trauma Center. J Trauma Acute Care Surg. 2011;71:69-77.

28. Mangram AJ, Mitchell CD, Shifflette VK, Lorenzo M, Truitt MS, Goel A, Lyons MA, Nichols DJ, Dunn EL. Geriatric trauma service: A one-year experience. J Trauma Acute Care Surg. 2012;72:119-22.

29. Bradburn E, Rogers FB, Krasne M, Rogers A, Horst MA, Belan MJ, Miller JA. High-risk geriatric protocol: Improving mortality in the elderly. J Trauma Acute Care Surg. 2012;73:435-40.

30. Stengel D, Rademacher G, Ekkernkamp A, Güthoff C, Mutze S. Emergency ultrasound-based algorithms for diagnosing blunt abdominal trauma. Cochrane Database Syst Rev. 2015;14:CD004446.

31. De Jongh MAC, van Stel HF, Schrijvers AJP, Leenen LPH, Verhofstad MHJ. The effect of helicopter emergency medical services on trauma patient mortality in the Netherlands. Injury. 2012;43:1362-7.

32. Galvagno Jr SM, Thomas S, Stephens C, Haut ER, Hirshon JM, Floccare D, Stephens C, Beecher D, Thomas S. Helicopter emergency medical services for adults with major trauma. Cochrane Database Syst Rev. 2015;12:CD009228.

33. Ringburg AN, Spanjersberg WR, Frankema SPG, Steyerberg EW, Patka P, Schipper IB. Helicopter emergency medical services (HEMS): impact on on-ScenetTimes. J Trauma Acute Care Surg. 2007;63:258-62.

34. Fukuma H, Nakada T, Shimada T, Shimazui T, Aizimu T, Nakao S, Watanabe H, Mizushima $Y$, Matsukoa T. Prehospital lactate improves prediction of the need for immediate interventions for hemorrhage after trauma. Sci Rep. 2019;9:13755.

\section{Figures}




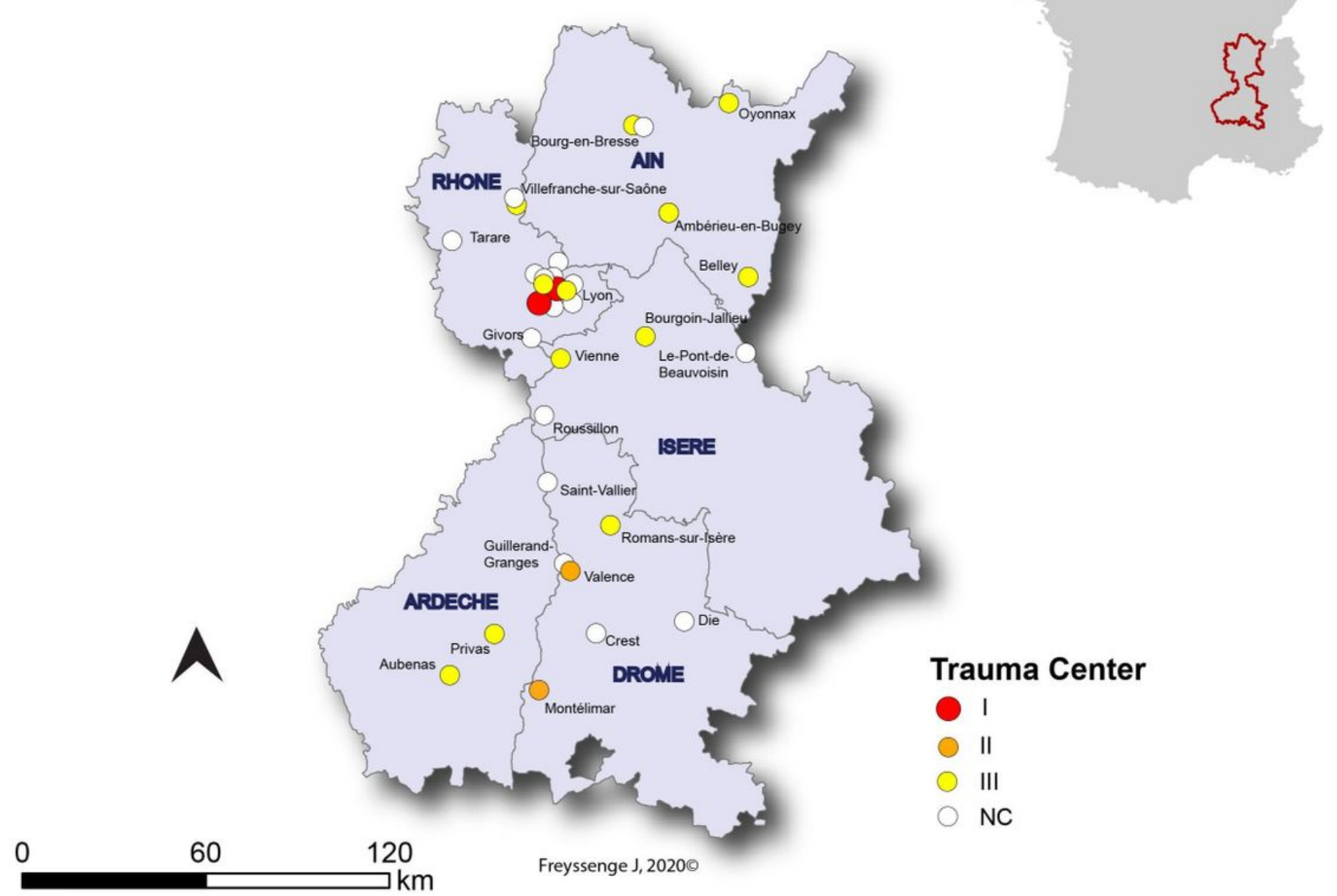

\section{Figure 1}

Trauma center in the study area Note: The designations employed and the presentation of the material on this map do not imply the expression of any opinion whatsoever on the part of Research Square concerning the legal status of any country, territory, city or area or of its authorities, or concerning the delimitation of its frontiers or boundaries. This map has been provided by the authors. 


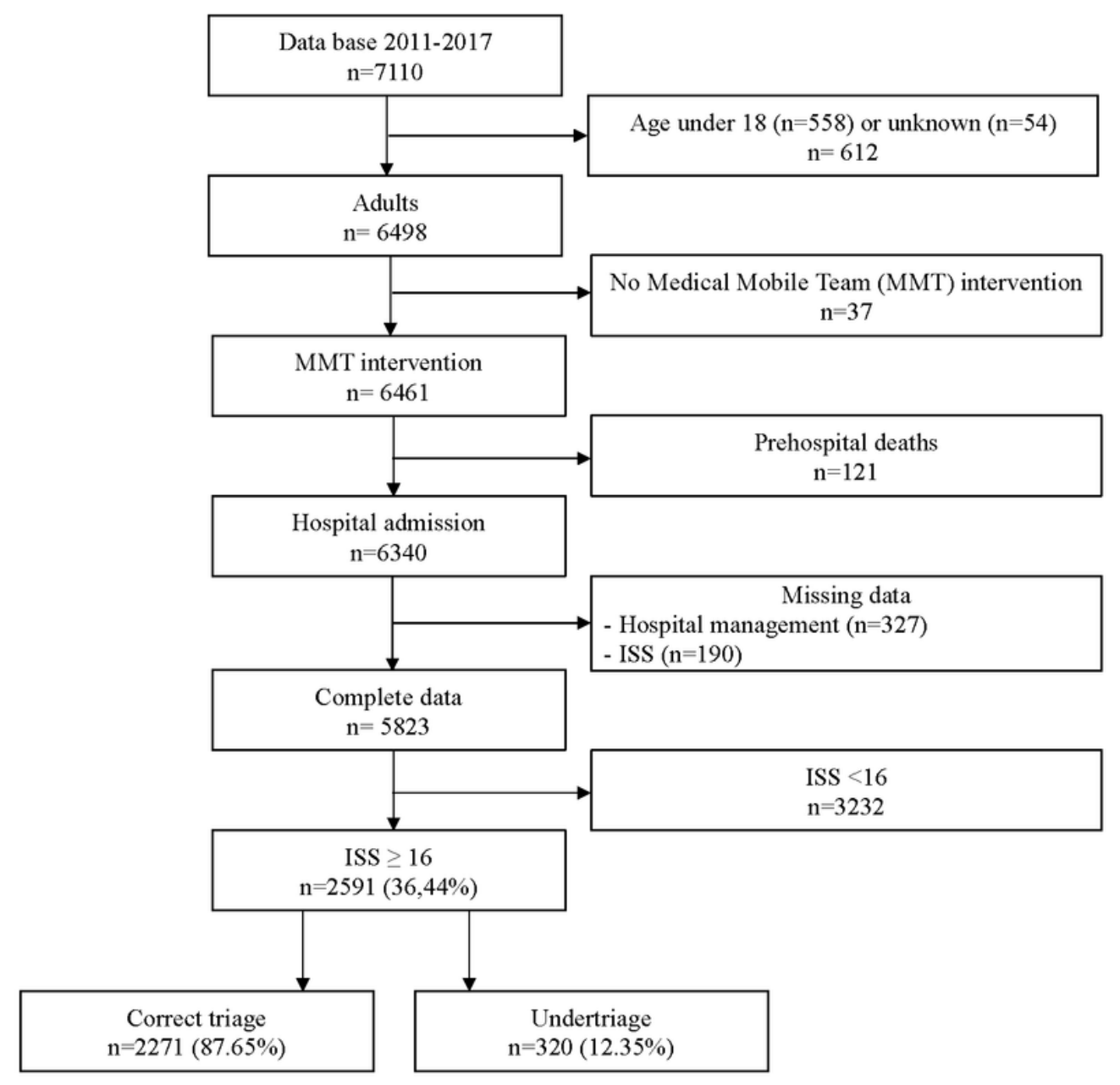

Figure 2

Flowchart of the study 
$\phi$ Univariate regression $\phi$ Multivariate regression

Epidemiological characteristics

Age class:

- 18-30 y.o (reference)

$-31-50$ y.o

$-51-65$ y.o

$-66-80$ y.o

-> 80 y.o

Sex : male

Season: winter

Year of inclusion

Cause of injury

- Road traffic injury (reference)

- Fall

- Gunshot/stab wounds

- Other

Penetrating injury

Pre-hospital timing and transport

On scene time ( $\mathrm{min})$

$-0-30 \mathrm{~min}$ (reference)

$-31-60 \mathrm{~min}$

- >60min

Transit time from scene

to hospital ( $\mathrm{min})$

$-0-15 \mathrm{~min}$ (reference)

$-16-30 \mathrm{~min}$

- >31 min

Air transport

Pre-hospital clinical data

Shock index $\geq 0.9$

Glasgow coma scale

- 15 (reference)

$-9-14$

$-\leq 8$

Pre-hospital management

Endotracheal intubation

FASTEcho

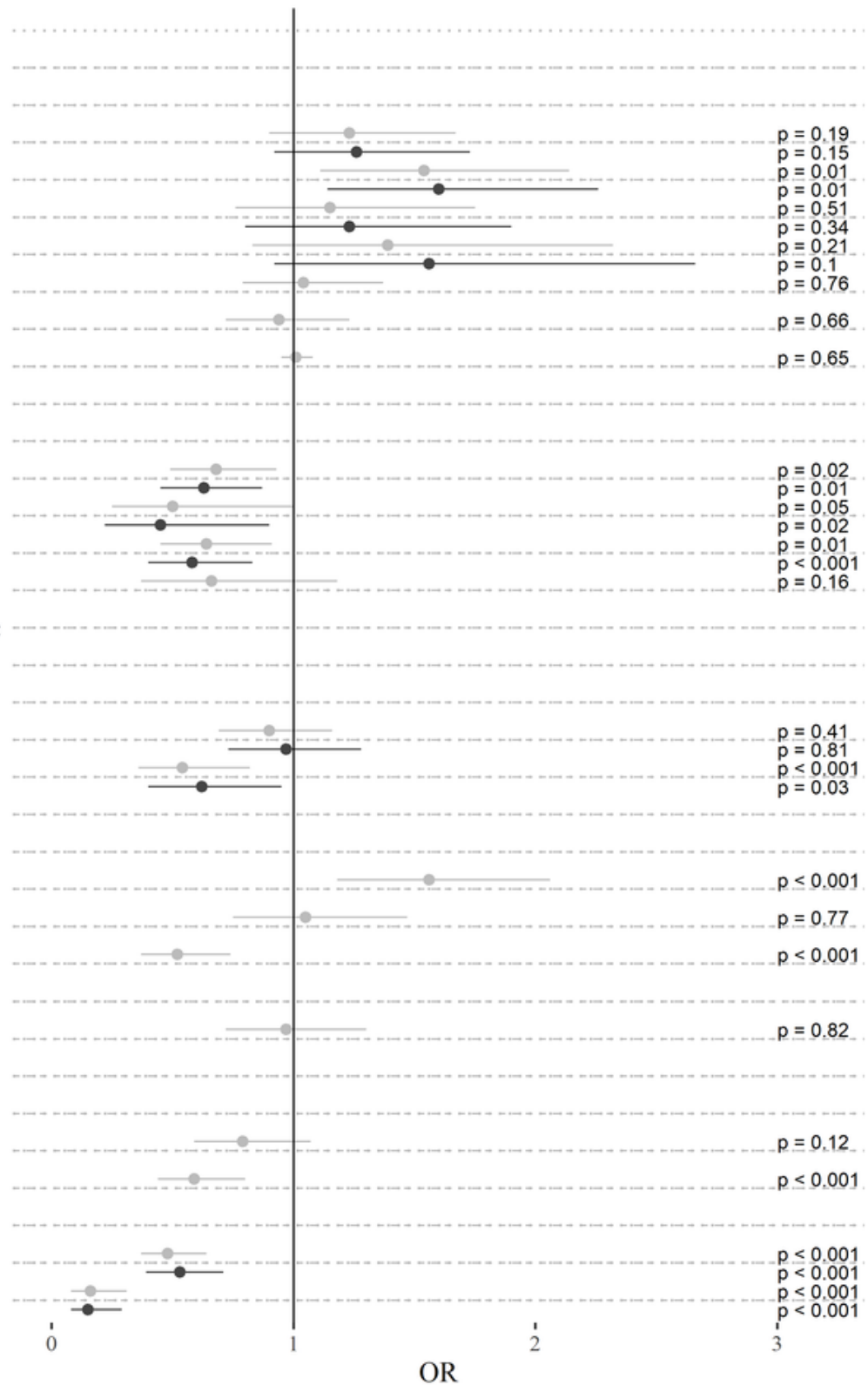

\section{Figure 3}

Determinants of undertriage 


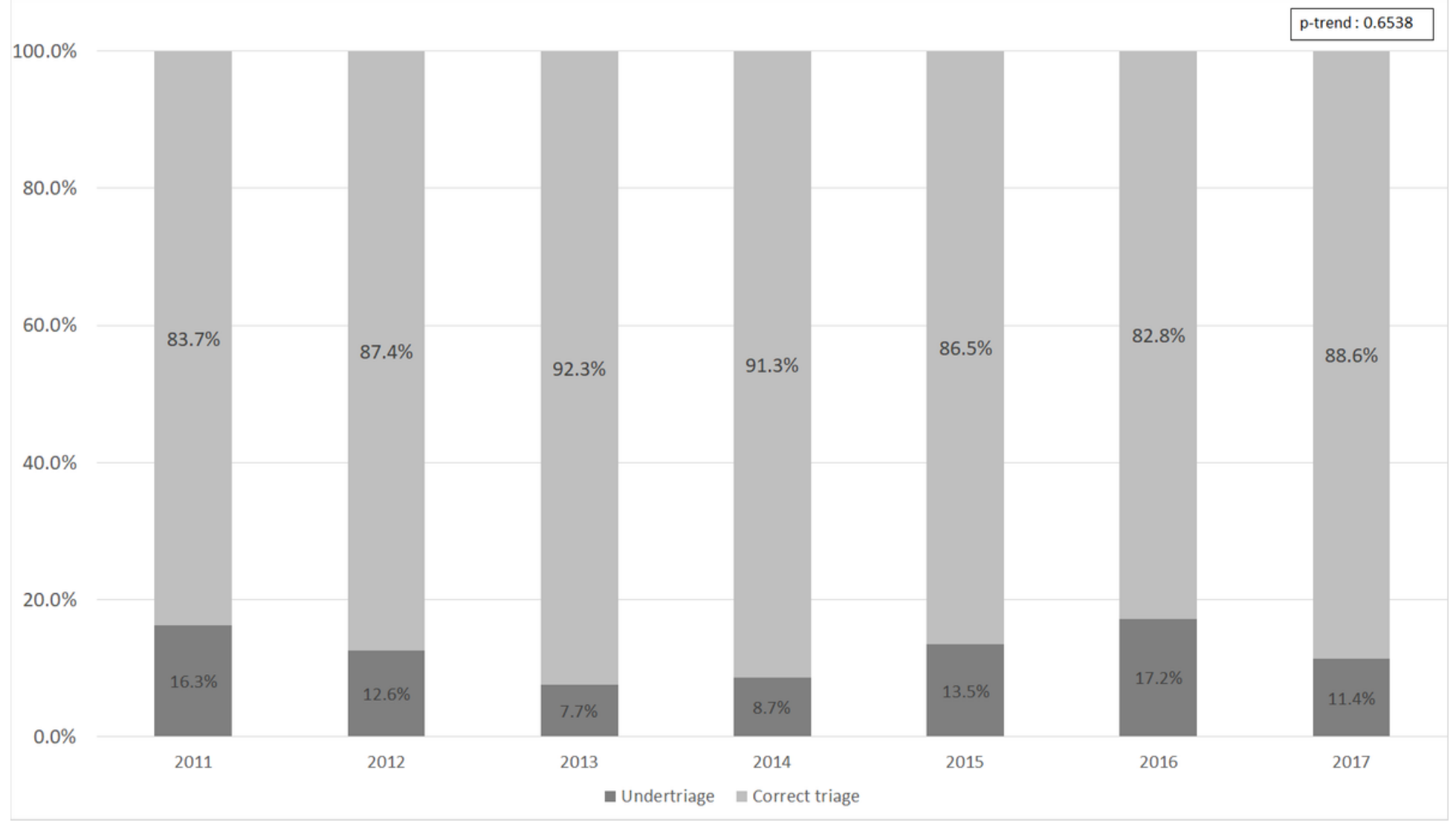

Figure 4

Annual evolution of undertriage

\section{Supplementary Files}

This is a list of supplementary files associated with this preprint. Click to download.

- Additionalfile1.docx

- Additionalfile2.pdf 